\title{
Materials-based Modelling of Packaging Effects on the Accuracy of Micro-Sensors
}

\author{
Erik Deier and Jürgen Wilde \\ University of Freiburg - IMTEK \\ Department of Microsystems Engineering \\ Laboratory for Assembly and Packaging \\ juergen.wilde@imtek.uni-freiburg.de
}

\section{SUMMARY}

In order to predict the performance of micro-systems in the development process, simulation has become an indispensable tool. Thermo-mechanical effects are among the strongest ones affecting the accuracy of micro-mechanical sensors. In this work, the procedures to predict the functional behaviour of microdevices correctly are demonstrated, taking into account packaging and assembly effects. The necessary models and data for a prediction of the thermo-mechanical behaviour are presented. The simulation accuracy depends substantially on the quality of the used materials data. Realistic results can only be achieved when time-temperature dependencies and non-linearity of the materials as well as correct process modelling are taken into account altogether. It will be demonstrated on the example of a pressure sensor how a new quality of prognosis will be possible when assembly and packaging are also regarded in the device design [1].

\section{SCOPE OF THE WORK}

When the silicon and the electronic circuit of a sensor are already optimised, the packaging is gaining more importance for the performance of the device. Features like costs, reliability, and size as well as sensitivity and accuracy must be optimised by selection of the appropriate packaging and interconnection technology, including design, materials and process variables [2], Fig. 1. Much of this work is still performed on an empirical basis although simulation is a well-established method to improve mechanical characteristics and reliability. Non-linear finite-element-simulation is already widely used in order to predict the lifetime of solder interconnects [3]. Up to now very few attempts have been made to utilise such methods to predict the functional behaviour of a micro-sensor device when it is subjected to thermal cycles in harsh environment. Therefore here a method is presented for the prognosis of a MEMS sensor's properties, which comprise sensitivity, accuracy and precision.

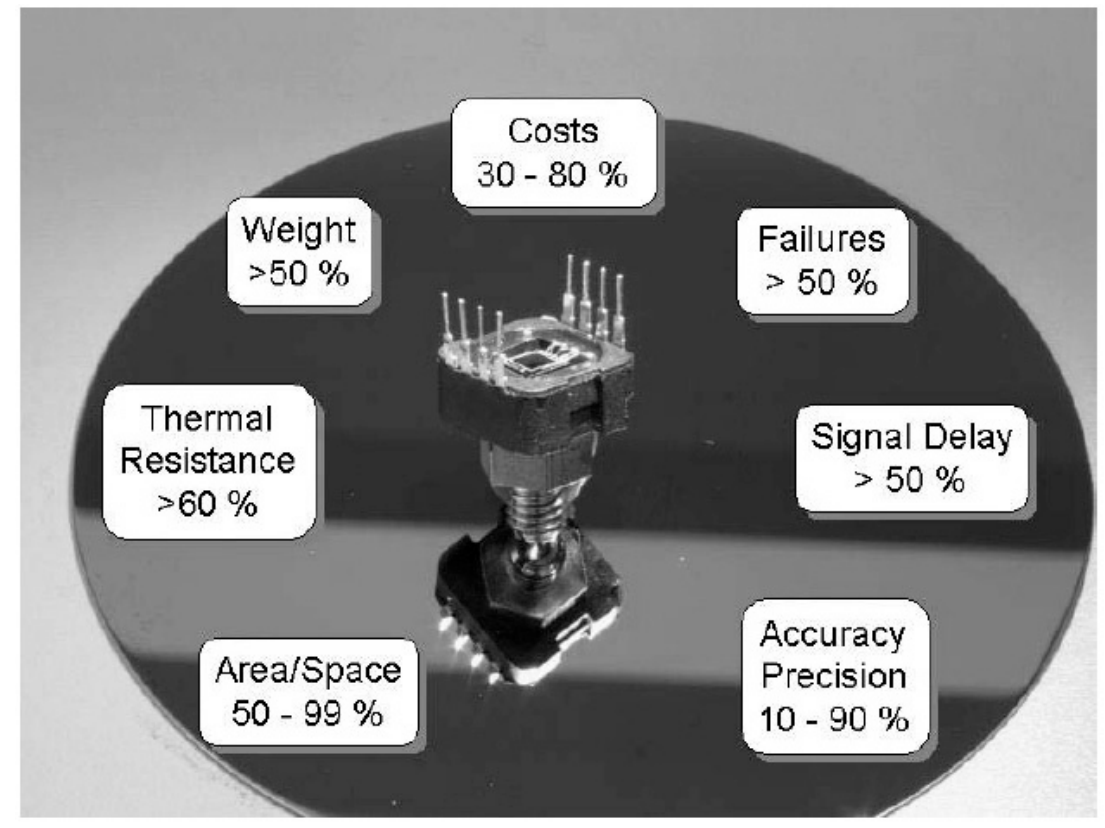

Figure 1: Relevant features of MEMS sensors which are affected by assembly and packaging. Data are based on the authors' own expertise. 


\section{EXPERIMENTAL WORK}

In the assembly and packaging process of MEMS sensors a variety of different materials are combined to mount, interconnect and seal the device. In the case regarded here a thermally conductive adhesives is used for the attachment of the MEMS sensor. The glue is applied to the substrate by dispensing. After placing the devices on the substrate, the adhesive is cured at temperatures between 80 and $175^{\circ} \mathrm{C}$.

The thermal mismatch problem is a typical effect of packaging and assembly technologies: When an assembly consisting of the sensor and the substrate is subjected to temperature changes, there will be a thermal expansion mismatch between the substrate and the chip. The mismatch can be expressed as a function of the temperature amplitude $\Delta T$ as well as of the thermal coefficients of expansion (TCE) $\alpha_{\text {Sub' }}$, $\alpha_{\mathrm{BE}}$ and the size of the device $\mathrm{I}_{0}$, Fig. 2:

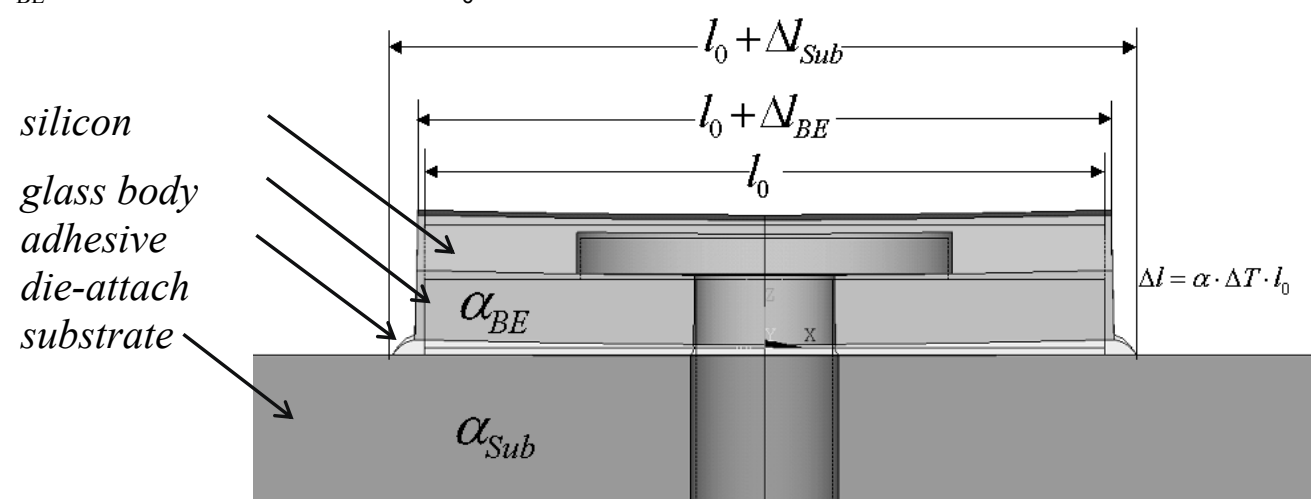

Figure 2: Thermal mismatch of an adhesively bonded silicon pressure sensor.

In our work, the influence of design and processes was analysed using the finite-element-method. An important initial step was the materials modelling of the thermo-mechanical properties of the die attachment, Fig. 3. Typically, a thermally conductive adhesive consists of an epoxy resin matrix, which is filled with ceramic particles or with silver flakes with a typical weight content of 70 to $85 \%$ (Ag).

In our work, thermal expansion, elasticity and visco-elasticity were modelled based on deformation measurements. Especially at elevated temperatures, epoxy materials show time-dependent materials behaviour. The performed tests on adhesives and epoxy moulding compounds exhibit that the polymers' mechanical behaviour is highly complex. Summarising, three mechanisms of strain deformation can occur in epoxy material at elevated temperatures namely elasticity, viscoelasticity and viscoplasticity:

$$
\gamma_{t o t}=\gamma_{e l}+\gamma_{v e l}+\gamma_{v p l} \text { or } \varepsilon_{t o t}=\varepsilon_{e l}+\varepsilon_{v e l}+\varepsilon_{v p l}
$$

In these formulas $\gamma$ and $\varepsilon$ denominate the shear strains and the normal strains respectively. Only in strain driven deformation problems, the viscoplastic deformation modes may be neglected for simulations. Then a Prony-series is appropriate for the mathematical description of the presented materials models, Fig. 4:

$$
G(t)=G_{\infty}+\sum_{i=1}^{n} G_{i} \cdot \exp \left(-\frac{t}{\phi_{i}}\right)
$$
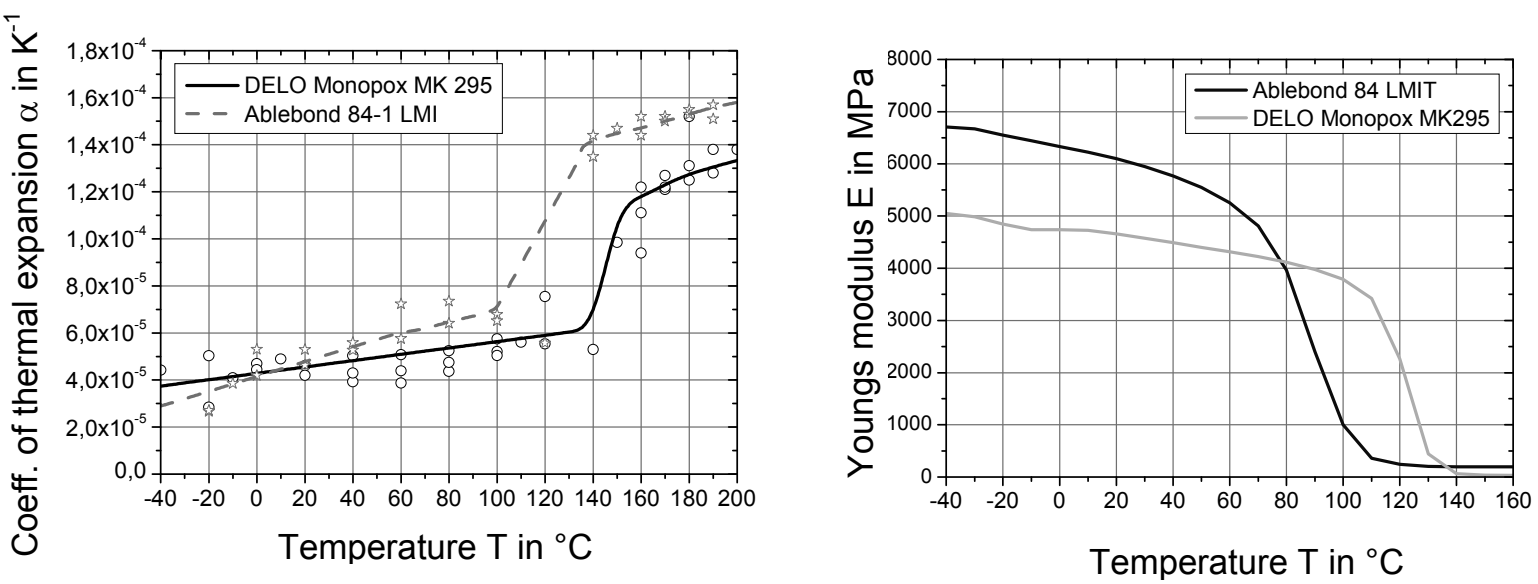

Figure 3: Coefficient of thermal expansion and Young's modulus of the adhesives analysed in this work. 

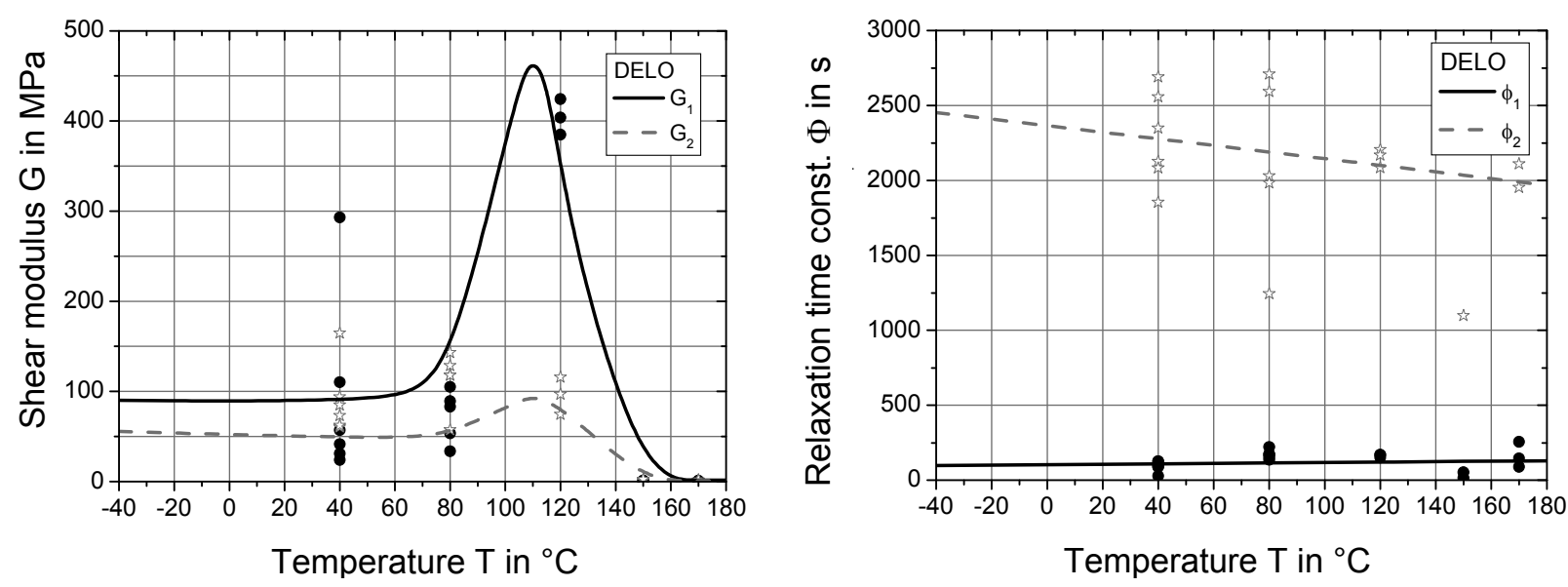

Figure 4: Viscoelastic shear-modulus and relaxation time constants for the ceramic-filled epoxy.

\section{RESULTS}

The next step was the FE analysis of a pressure sensor's behaviour. In order to predict the sensors' characteristics, finite-element-models were designed which comprise the relevant components like substrate or housing, die attachment, glass body and silicon device. In the computations, different heating cycles from $20^{\circ} \mathrm{C}$ up to $150{ }^{\circ} \mathrm{C}$ with successive hold times and subsequent cooling back to ambient were assumed. The radial mechanical stress near the circumference of the membrane was used as a criterion because this property has direct influence on the sensor signal. The selected position is where typically the stress-sensing structures are located on the sensor, Fig. 5. Also the thermo-mechanical properties of the assembly materials of a MEMS pressure gauge were varied.

One can regard the mechanical stress in the membrane as a cause for the offset signal of the sensor. In one of the analysed examples, the evolution of temperature-dependent stress will lead to an increase when the temperature rises. Also the stress results were further processed numerically in order to compute the electrical behaviour of the sensor devices. A basic dependency is the proportionality of mechanical stress $\sigma$ and the piezo-resistance change.

Two strong parameters affecting the qualitative and quantitative behaviour of the stress history over temperature cycles are the curing temperature and the glass transition temperature: The curing temperature and the transient temperature gradient in the cured adhesive determine the stress-free temperature in the assembly. A reasonable assumption is a stress-free temperature close to the curing temperature as this is the state where the cross-links in the adhesive are created. Caused by the curing shrinkage of the adhesive, a stress-free temperature, which is slightly higher than the curing temperature can be expected.
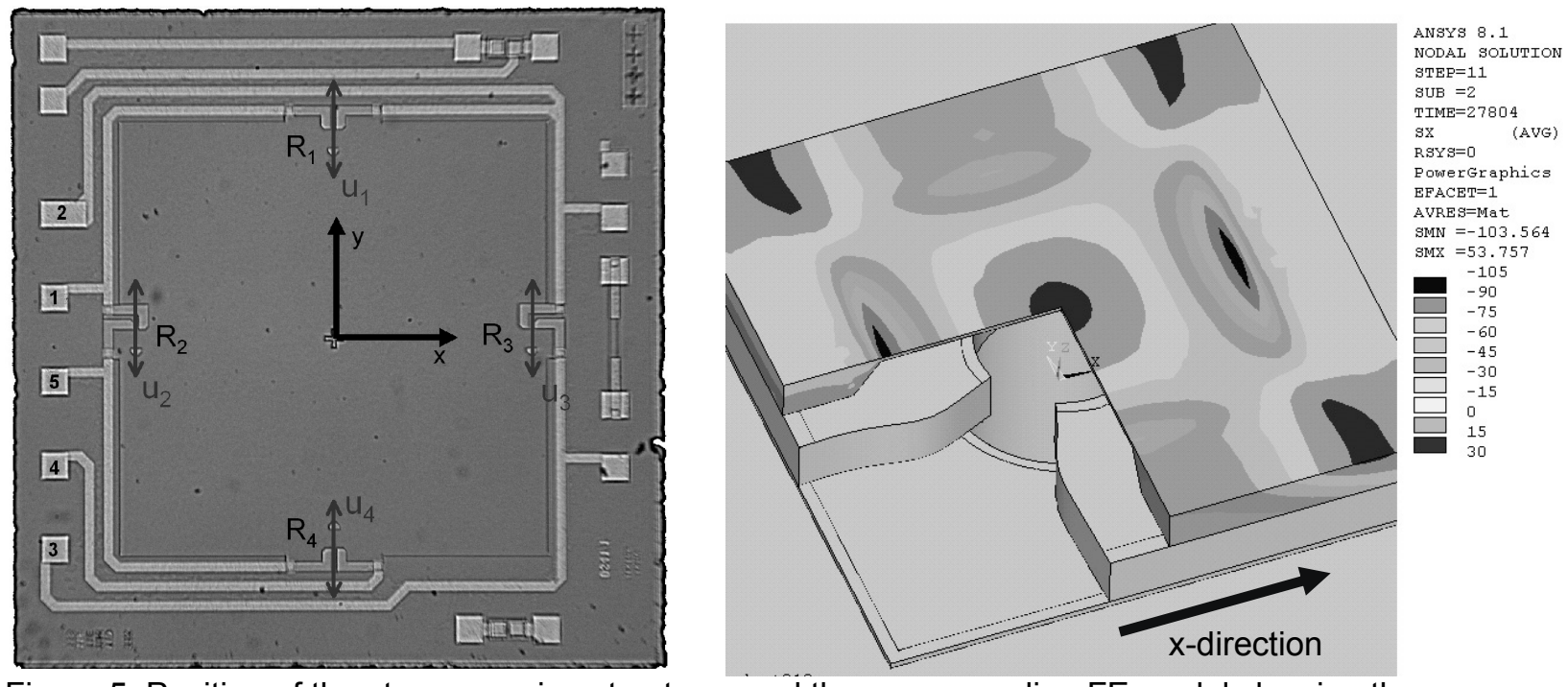

Figure 5: Position of the stress-sensing structures and the corresponding FE model showing the $x$ component of the membrane stress at a pressure $p=0.3$ bar and a temperature of $40{ }^{\circ} \mathrm{C}$. 
With a slow heating gradient in the curing process, the first cross-links in the adhesive will build up before the actual curing temperature is reached. This effect will shift the stress free state to lower temperatures. So does long storage time at ambient temperatures as relaxation processes will occur. In this work we could show that the stress-free temperature in combination with the glass transition temperature has a significant influence on the evolution of thermo-mechanical stresses over temperature, Fig. 6 (right). For low stress-free temperatures $T_{\text {ref }}$ the calculated zero-signal is ascending with rising temperature. It reaches a maximum near the glass transition temperature. As the adhesive is softening, the zero signal approaches the zero point.

For stress-free temperatures above the glass transition temperature, a decline of the gradient is discovered at the glass transition temperature. This leads to completely different types of zero-point signals over temperature. We also discovered a very similar behaviour in our experiments on real sensor assemblies, , Fig. 6 (left). Therefore the simulations were performed on models of industrial MEMS pressure elements, which represented precisely the assembled sensors with respect to substrates and packages. With the correct choice of the stress-free temperature and the time-dependent deformation model for the adhesive, the non-linear output behaviour of the sensor during thermal cycling was both measured and modelled with a very good agreement, Fig. 6 (left).

It is also evident, that a variation of the curing temperature and therefore the stress-free temperature $T_{\text {ref }}$ will not only affect thermal strains proportionally via the $\Delta T \Delta \alpha$-term. $T_{\text {ref }}$ will also have a strong qualitative effect on the stress and the offset of a device over the operation temperature. Therefore a sensor's behaviour will be significantly affected if it's die attachment is not fully hardened during fabrication and if it post-hardens during storage or operation.
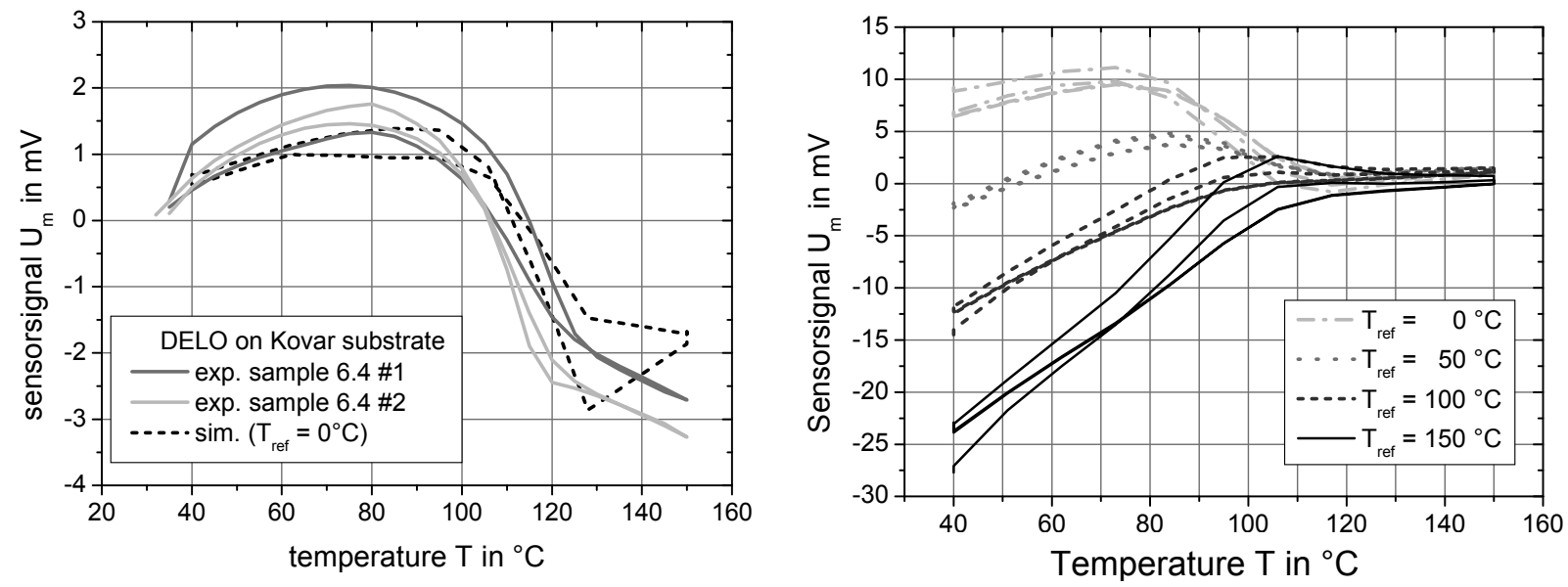

Figure 6: Measured temperature-dependent output signal (zero point) of a pressure sensor in comparison with simulation results (left). Influence of the stress-free temperature $T_{\text {ref }}$ on the shift of zero point over temperature. Assembly with Ablebond adhesive on steel substrate (right).

Up to now, the stress-free state of an assembly cannot be modelled in advance. Experimental methods like measuring deflection of adhesively joined systems are limited to easy geometries with not more than one material with time dependent deformation mechanism. Current work with stress sensing chips evaluated during their packaging process will overcome these issues $[4,5,6]$

\section{CONCLUSIONS}

In this paper we have presented a concept how the properties of MEMS-based sensors can be predicted and optimised when the packaging has strong effects on the thermo-mechanical behaviour. The concept is represented graphically in Figure 7.

1. The principal tool of the design flow is a commercial multi-physics finite-element-program. In a first step of the analysis a detailed FE model is created.

2. The geometry model is combined with the materials data of the complete package. These data must take into account the temperature dependency, the time dependency and the non-linearity of the materials. This is an indispensable prerequisite for correct physical modelling. 
3. Based on these inputs the life-cycle history will be simulated. This analysis has to reproduce the relevant time-temperature profiles of the manufacturing and the operational phases.

4. As a result stresses and strains, which are induced in the functional structure of the MEMS, will be computed.

5. By analysis of these loads the functional properties of the device like offset and sensitivity can be computed. To that purpose a physical model, which describes sensitivity and cross-sensitivities of the MEMS device must be available.

6. In order to verify the correctness of the prognosis model testing of representative samples is helpful. Based on these results it was shown, that good qualitative and quantitative matching of the simulation results and of the test work is possible

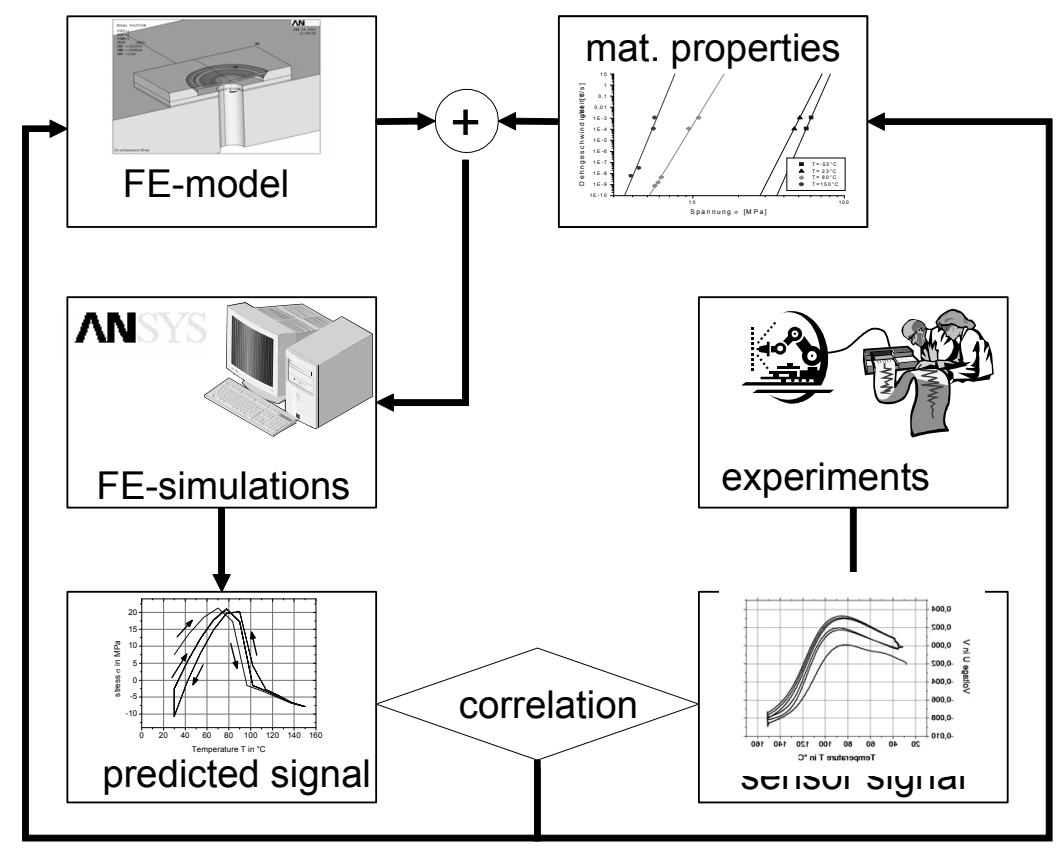

Fig. 7: Concept for the simulation-based design optimisation of industrial MEMS pressure sensors

\section{REFERENCES}

[1] Deier, E.; Dissertation, Universiy of Freiburg, 2008. Der Andere Verlag, Tönning 2008

[2] Wilde, J.: Materials-based modelling of non-linear mechanical MEMS polymers. Multiscale Materials Modeling, Third International Conference, Freiburg, Germany, 18 - 22 September 2006

[3] Dudek R. et al: Simulation based analysis of secondary effects on solder fatigue, Proceeding EuroSimE 2008, Freiburg

[4] Kittel, H.: Sensor-IC zur Detektion verpackungsbedingter Stresseinträge auf Mikrosysteme. Proceedings Sensor und Test, Nuremberg 2006

[5] Doelle, M. et al: Geometry optimization for planar piezoresistive stress sensors based on the pseudo-Hall effect. Sensors and Actuators A 127, 2006

[6] Pustan, D., Wilde, J.: Modern test methods for a comprehensive thermo-mechanical deformation analysis in area-array-assemblies. 58th Electronic Components \& Technology Conference, Orlando, Florida, USA, 27 - 30 May 2008

\section{AFFILIATION}

Dr.-Ing. Jürgen Wilde, Professor

Department of Microsystems Engineering - IMTEK

University of Freiburg

Georges-Koehler-Allee 103

79110 Freiburg, Germany

Phone: +49 761-203 7291, -7290

Fax: +49 761-203 7292

Email: juergen.wilde@imtek.uni-freiburg.de

URL: http://www.imtek.de/avt/english/ 